\title{
Phenytoin, Levetiracetam, and Pregabalin in the Acute Management of Refractory Status Epilepticus in Patients with Brain Tumors
}

\author{
Christa B. Swisher • Meghana Doreswamy • \\ Krista J. Gingrich · James J. Vredenburgh • \\ Brad J. Kolls
}

Published online: 1 September 2011

(C) Springer Science+Business Media, LLC 2011

\begin{abstract}
Background There were nearly 700,000 patients in the United States in 2010 living with brain tumor diagnoses. The incidence of seizures in this population is as high as $70 \%$ and is historically difficult to control. Approximately $30-40 \%$ of brain tumors patients who present with status epilepticus (SE) will not respond to typical therapy consisting of benzodiazepines and phenytoin (PHT), resulting in patients with refractory status epilepticus (RSE). RSE is usually treated with anesthetic doses of propofol or midazolam infusions. This therapy can have significant risk, particularly in patients with cancer.

Methods A retrospective chart review was performed on 23 patients with primary or metastatic brain tumors whose SE was treated with intravenous PHT, levetiracetam (LEV), and oral pregabalin (PGB).

Results In all the patients under study, PHT or LEV was used as first-line therapy. PGB was typically used as thirdline treatment. The median daily dose of PGB was $375 \mathrm{mg}$ (usually divided BID or TID), and the median daily dose of LEV $3000 \mathrm{mg}$ (usually divided BID). Cessation of SE was seen in 16/23 (70\%) after administration of PHT, LEV, and PGB. SE was aborted, on average, $24 \mathrm{~h}$ after addition of the third antiepileptic drug. Only one patient in the responder group required intubation. Mortality rate was zero in the responder group. No adverse reactions to this medication regimen were observed.
\end{abstract}

C. B. Swisher · M. Doreswamy · K. J. Gingrich .

J. J. Vredenburgh · B. J. Kolls ( $\square)$

Division of Neurology, Department of Medicine,

Duke University Medical Center (DUMC),

Box 2900, Durham, NC 27710, USA

e-mail: kolls001@mc.duke.edu
Conclusion Our study suggests that the administration of PHT, LEV, and PGB in brain tumor patients with RSE is safe and highly effective.

Keywords Refractory status epilepticus . Status epilepticus - Brain tumor · Phenytoin · Levetiracetam $\cdot$ Pregabalin

\section{Background and Significance}

In the United States in 2010, there was an estimated population of 6,88,096 individuals living with primary brain tumor diagnoses, and the prevalence rate for these tumors was estimated to be 221.8 per $1,00,000$ [1] Seizures in patients with primary and metastatic brain tumors occur commonly. Previous studies have shown that the incidence of seizures in patients with brain tumors is $30-70 \%[2,3]$. Seizures are notoriously difficult to control in the brain tumor population. Hildebrand et al. [4] found that over half of brain tumor patients continued to have seizures despite treatment with AEDs. In a study by Moots et al. [5] 26\% of patients with brain tumors and epilepsy developed status epilepticus (SE). Furthermore, up to $20 \%$ of adult patients with SE have brain tumors as the cause of SE [6-11]. The overall mortality rate from $\mathrm{SE}$ of all causes remains high at $15-23 \%[6,7,11]$; however, the mortality is even higher, $30-40 \%$, in brain tumor patients $[7,11,12]$. It has been reported that only $60-70 \%$ of SE in brain tumor patients is aborted by benzodiazepines and phenytoin (PHT) [13, 14], resulting in a significant number of patients with refractory status epilepticus (RSE). When initial management fails to control the seizures, typically anesthetic agents such as propofol and midazolam are used to place the patient into an iatrogenic coma. This therapy requires intubation and 
mechanical ventilation. Not only are brain tumor patients more susceptible to ventilator-associated complications such as pneumonia due to immunosuppression from chemotherapy, but also many patients have advanced directives which have to be reversed or altered to allow this level of intervention to be undertaken. This can lead to family stress and conflicts during therapy, particularly when the seizures remain after treatment.

Non-sedating, newer generation antiepileptic drugs (AEDs) such as lacosamide, pregabalin, and topirimate are being investigated to treat RSE to avoid the complications of mechanical ventilation. There is good data for PHT and levetiracetam (LEV) in the treatment of RSE [13-18]. Pregabalin (PGB) is only available as an oral agent, but it is considered as a potential option in treating RSE since it has a good safety profile, can be started at a high dose, does not interact with other AEDs and has good oral bioavailability. A preliminary study by Novy and Rossetti [19] showed that 5/11 patients with RSE demonstrated a clinical response to the addition of PGB after the patients were treated with a benzodiazepine and another AED. PGB has a different mechanism of action than PHT or LEV. PGB inhibits the alpha-2-delta protein of the presynaptic calcium channel, while PHT blocks voltage-sensitive sodium channels and LEV binds to the synaptic vesicle protein 2A [20-22].

There are currently no guidelines for the treatment of brain tumor patients with RSE. The aim of this study was to provide evidence for a treatment regimen that does not require intubation. Patients with primary and metastatic brain tumors admitted to our institution with SE have been treated with the combination PHT, LEV, and PGB to avoid over-sedation and intubation of patients. We have used the phrase "the Trifecta" to describe the use of these three AEDs together.

\section{Methods}

Permission to complete the study was first obtained from the Duke Institutional Review Board. Initial patient identification and selection was performed using the DEDUCE (Duke enterprise data unified content explorer) database. This is an on-line query system based on data collected into the decision support repository (DSR) from operational systems serving each of the Duke hospitals. Query criteria included patients who were diagnosed as having a brain tumor, presented with complex partial SE between January 2006 and December 2009 and received the Trifecta (PHT, LEV, and PGB). Patients that were found in the database to meet all the three of these criteria were then reviewed further manually to determine appropriateness for the study. No patients under 18 were included. Target total serum PTH level was $15-25 \mu \mathrm{g} / \mathrm{ml}$.
SE was defined as repeated clinical or electrographic seizures without full recovery between seizures for longer than $30 \mathrm{~min}$. Clinical and demographic data were collected from our hospital's electronic medical record system. A clinical response to treatment with the Trifecta was defined as complete resolution of clinical seizures and/or cessation of repetitive epileptiform discharges on EEG, if no other AEDs were added during hospitalization and if SE did not recur during hospitalization. The 1 year outcomes were collected from the electronic medical record. Statistical analysis was performed using Student's $t$-test for nominal variables and chi-squared analysis for categorical variables.

\section{Results}

From the DEDUCE database, we retrospectively identified 8,493 brain tumor patients that were seen between January 2006 and December 2009. Of these patients, 2,606 (31\%) patients were diagnosed with seizures. From this group of 2,606 patients, 539 (21\%) were on more than one AED to control their seizures. We found 56 of these patients were identified as having received the Trifecta (PHT, LEV, and PGB). Brief manual review of these 56 patient records resulted in identification of 23 patients that met all the inclusion criteria. A more detailed retrospective chart review as well as EEG analysis was then performed on these 23 patients.

Overall clinical features extracted from the chart are provided in Table 1 . The average age was 56.9 years old (range 25-84), and there were approximately equal numbers of males and females. $70 \%$ of patients had a prior history of seizures. There was tumor progression in $26 \%$ of patients at the time of diagnosis of RSE. The percentage of patients having undergone resection for their brain tumor was $91 \%$. Thirty-nine percent of patients received radiation, and another 39\% received chemotherapy. About half of the patients (52\%) underwent biopsy or resection of their brain tumor within 1 month of SE onset. Nearly all of the patients $(91 \%)$ were on an anticonvulsant at the time of diagnosis of RSE. The majority of patients $(61 \%)$ presented with obvious SE (abnormal, repetitive motor movements of the face, leg, or arm). The rest presented with subtle SE which included signs and symptoms, such as aphasia, altered mental status, focal weakness, and lip smacking. The most common tumor type was glioblastoma multiforme (52\%). Tumor location was highly variable, with temporal lobe being the most common location followed by frontal lobe tumors.

There was a trend toward the presence of peritumoral edema on MRI being associated with response to the Trifecta. Four patients in the responder group had 
Table 1 Patient characteristics

\begin{tabular}{|c|c|c|c|c|}
\hline & All & Responders & Non-responders & $P$ value \\
\hline Number of patients & 23 & 16 & 7 & \\
\hline Average age & $56.9 \pm 15.9$ & $56.4 \pm 16.3$ & $58 \pm 16$ & 0.83 \\
\hline Male gender & $11(47.8 \%)$ & $9(56 \%)$ & $2(29 \%)$ & 0.22 \\
\hline \multicolumn{5}{|l|}{ Treatment: } \\
\hline Underwent resection & $21(91 \%)$ & $14(88 \%)$ & $7(100 \%)$ & 0.33 \\
\hline Chemotherapy & $9(39 \%)$ & $6(38 \%)$ & $3(43 \%)$ & 0.81 \\
\hline Radiation & $9(39 \%)$ & $3(19 \%)$ & $4(57 \%)$ & 0.24 \\
\hline Biopsy or resection within the past month & $12(52 \%)$ & $9(56 \%)$ & $3(43 \%)$ & 0.55 \\
\hline $\begin{array}{l}\text { Average time from biopsy or resection to onset of status } \\
\text { epilepticus (SE) (days) }\end{array}$ & $202 \pm 279$ & $149 \pm 234$ & $323 \pm 351$ & 0.17 \\
\hline Prior history of seizures & $16(70 \%)$ & $10(63 \%)$ & $6(86 \%)$ & 0.27 \\
\hline Stable tumor status & $17(74 \%)$ & $12(75 \%)$ & $5(71 \%)$ & 0.86 \\
\hline On decadron at time of SE onset & $15(65 \%)$ & $11(69 \%)$ & $4(57 \%)$ & 0.59 \\
\hline On an anticonvulsant at time of SE onset & $21(91 \%)$ & $16(100 \%)$ & $5(71 \%)$ & 0.03 \\
\hline On PHT at baseline & $6(26 \%)$ & $5(31 \%)$ & $1(14 \%)$ & 0.39 \\
\hline On LEV at baseline & $14(61 \%)$ & $10(63 \%)$ & $4(57 \%)$ & 0.81 \\
\hline On PHT and LEV at baseline & $2(9 \%)$ & $1(6 \%)$ & $1(14 \%)$ & 0.53 \\
\hline On PGB at baseline & 0 & 0 & 0 & \\
\hline $\begin{array}{l}\text { Presentation subtle } \\
\text { (aphasia, confusion, focal weakness, lip smacking) }\end{array}$ & $9(39 \%)$ & $6(36 \%)$ & $3(43 \%)$ & 0.81 \\
\hline Presentation obvious (face or extremity twitching) & $14(61 \%)$ & $10(63 \%)$ & $4(57 \%)$ & 0.81 \\
\hline Edema present & $4(19 \%)$ & $4 / 14(29 \%) *$ & 0 & 0.12 \\
\hline Hemorrhage present & $8(38 \%)$ & $5 / 14(36 \%) *$ & $3 / 7(42.8 \%)$ & 0.75 \\
\hline \multicolumn{5}{|l|}{ Tumor grade } \\
\hline WHO I & $1(4 \%)$ & $1(6 \%)$ & 0 & 0.50 \\
\hline WHO II & $6(26 \%)$ & $3(19 \%)$ & $3(43 \%)$ & 0.23 \\
\hline WHO III & $2(9 \%)$ & $2(13 \%)$ & 0 & 0.33 \\
\hline WHO IV & $12(52 \%)$ & $9(39 \%)$ & $3(43 \%)$ & 0.55 \\
\hline Metastatic lesion & $2(9 \%)$ & $1(6 \%)$ & $1(14 \%)$ & 0.53 \\
\hline
\end{tabular}

* Two patients did not have imaging

peritumoral edema on MRI, compared to nil patients in the non-responder group. However, this was not statistically significant $(P=0.12)$. More patients in the responder group were on an AED at baseline (100\% vs. $71 \%)$, and this was statistically significant $(P=0.03)$. Periodic lateralized epileptiform discharges (PLEDs) were a common interictal finding in both groups.

The clinical outcomes are shown in Table 2. In all patients, PHT or LEV was used as first-line therapy. PGB was typically used as third-line treatment. In some of the cases (7/23), PGB was used as second-line therapy. The majority of cases were treated with LEV followed by PHT followed by PGB when electrographic or clinical SE was still ongoing. The median daily PGB dose was $375 \mathrm{mg}$ (usually divided BID or TID). PGB was administered via nasogastric tube or orally. PHT and LEV were administered intravenously. The median daily LEV dose was $3,000 \mathrm{mg}$ daily (usually divided BID). For the patients that responded to the Trifecta, the average PHT blood level at time of cessation of SE was 18.9. Blood levels of LEV and PGB were not routinely measured.

In the responder group, 16/23 (70\%) patients had cessation of RSE after administration of the third component of the Trifecta. The length of RSE in the responders was 3.5 days on average. Figure 1 shows the percentage of patients still in RSE over a period of time. The average time it took to administer the third-line treatment was 2.1 days. The average time it took for RSE to stop after administration of the third-line agent was approximately $24 \mathrm{~h}$.

In $7 / 23$ (30\%) patients, seizures did not cease after the administration of the Trifecta. Of these seven patients, five of them eventually had cessation with the addition of valproic acid (2 patients), topiramate (1 patient), or midazolam infusion (2 patients). There was a statistically significant difference in mortality rate $(0 \%$ in the responder 
Table 2 Clinical outcomes

\begin{tabular}{|c|c|c|c|c|}
\hline & All patients & Responders & Non-responders & $P$ value \\
\hline $\begin{array}{l}\text { Average time from SE onset to administration } \\
\text { of third AED (days) }\end{array}$ & $2.3 \pm 1.5$ & $2.3 \pm 1.6$ & $2.1 \pm 1.7$ & 0.81 \\
\hline Average length of SE (days) & $3.6 \pm 2.0$ & $3.5 \pm 1.6$ & $4.2 \pm 2.9 * *$ & 0.46 \\
\hline Intubated & $4(17 \%)$ & $1(6 \%)$ & $3(43 \%)$ & 0.03 \\
\hline Died & $2(9 \%)$ & 0 & $2(29 \%)$ & 0.03 \\
\hline \multicolumn{5}{|l|}{ EEG pattern } \\
\hline Focal seizures & $6(26 \%)$ & $3(19 \%)$ & $3(43 \%)$ & 0.23 \\
\hline Secondarily generalized & $3(13 \%)$ & $2(13 \%)$ & $1(14 \%)$ & 0.91 \\
\hline PLEDs + focal seizures & $10(44 \%)$ & $9(56 \%)$ & $1(14 \%)$ & 0.60 \\
\hline PLEDs + secondarily generalized seizures & $3(13 \%)$ & $1(6 \%)$ & $2(29 \%)$ & 0.14 \\
\hline Clinical (No EEG) & $1(4 \%)$ & $1(6 \%)$ & 0 & 0.50 \\
\hline \multicolumn{5}{|l|}{ Order of AEDs } \\
\hline PHT/LEV $\rightarrow$ PGB & $2(9 \%)$ & $1(6 \%)$ & $1(14 \%)$ & 0.53 \\
\hline $\mathrm{LEV} \rightarrow \mathrm{PHT} \rightarrow \mathrm{PGB}$ & $12(52 \%)$ & $7(44 \%)$ & $5(71 \%)$ & 0.22 \\
\hline $\mathrm{PHT} \rightarrow \mathrm{LEV} \rightarrow \mathrm{PGB}$ & $5(22 \%)$ & $5(31 \%)$ & 0 & 0.09 \\
\hline $\mathrm{LEV} \rightarrow \mathrm{PGB} \rightarrow \mathrm{PHT}$ & $3(13 \%)$ & $2(13 \%)$ & $1(14 \%)$ & 0.91 \\
\hline $\mathrm{PHT} \rightarrow \mathrm{PGB} \rightarrow \mathrm{LEV}$ & $3(13 \%)$ & $3(19 \%)$ & 0 & 0.22 \\
\hline Average LEV daily dose (mg) & $3391 \pm 916$ & $3406 \pm 970$ & $3357 \pm 852$ & 0.91 \\
\hline Average PGB daily dose (mg) & $421 \pm 151$ & $378 \pm 152$ & $517 \pm 116$ & 0.16 \\
\hline Average PHT level at time SE stopped $(\mathrm{mcg} / \mathrm{ml})$ & $18.8 \pm 5.2$ & $18.9 \pm 4.5$ & $18.3 \pm 7.8^{* *}$ & 0.89 \\
\hline \multicolumn{5}{|l|}{ AEDs on discharge } \\
\hline $\mathrm{PHT}+\mathrm{LEV}+\mathrm{PGB}$ & $15 / 2171 \%)$ & & & \\
\hline $\mathrm{LEV}+\mathrm{PGB}+\mathrm{VPA}$ & $2 / 21(10 \%)$ & & & \\
\hline $\mathrm{PHT}+\mathrm{PGB}$ & $2 / 21(10 \%)$ & & & \\
\hline $\mathrm{PHT}+\mathrm{LEV}+\mathrm{PGB}+\mathrm{VPA}$ & $1 / 21(5 \%)$ & & & \\
\hline $\mathrm{LEV}+\mathrm{PHT}$ & $1 / 21(5 \%)$ & & & \\
\hline
\end{tabular}

** Two patients did not have cessation of RSE and were not included in this part of the analysis

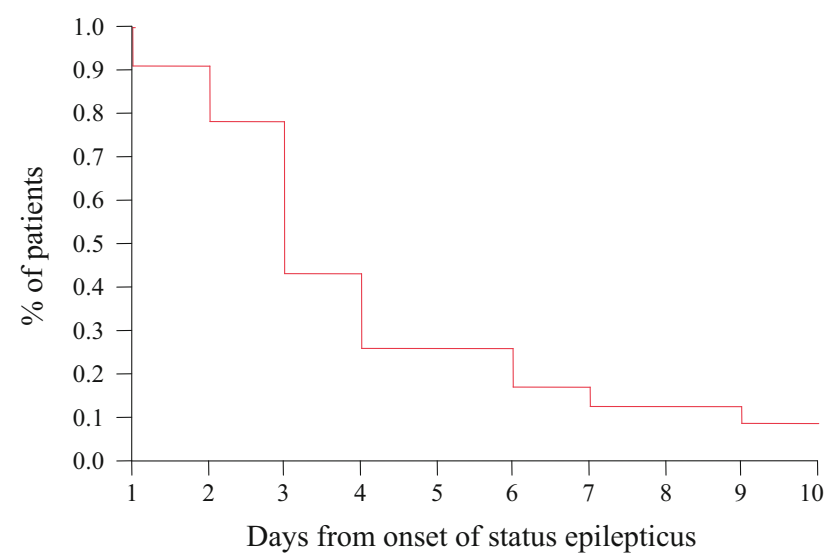

Fig. 1 Duration of status epilepticus (SE) demonstrates the percentage of patients remaining in SE over time. The average time it took to administer the third-line AED was 2.1 days. The average time it took for RSE to stop after administration of the third-line AED was approximately $24 \mathrm{~h}$

group and $29 \%$ in the non-responder group, $P=0.03$ ). Two of the seven patients that did not respond to the Trifecta died. One patient had persistent seizures resulting in somnolence. Midazolam infusion was attempted, but did not result in cessation of seizures. The family decided to withdraw care. The other patient that died had persistent seizures on EEG which resulted in unresponsiveness and bradycardia. Owing to this and tumor progression on MRI, the family withdrew care.

There was a statistically significant difference in intubation rates (1/16 patients in the responder group and 3/7 patients in the non-responder group, $P=0.03$ ). Two of the three intubated patients in the non-responder group were placed on a midazolam infusion in an attempt to abort RSE.

Upon discharge from the hospital, 15 of the 21 patients that were alive were discharged on the Trifecta. At 1 year follow-up, eight $(35 \%)$ patients died, six $(26 \%)$ had radiographic progression, eight $(35 \%)$ were clinically and radiographically stable, and one patient was lost to followup. Most patients were on monotherapy at 1 year follow-up. Eighty-three percent of patients were on LEV, $67 \%$ of patients were on PGB, and $42 \%$ of patients were on PHT at the 1 year follow-up visit. Only a minority of patients $(2 / 15)$ were still on all the three AEDs. 


\section{Discussion}

We have found that $31 \%$ of the brain tumor patients under this study have seizures, and that nearly a quarter of these patients require more than 1 AED to control their seizures. This is consistent with previous studies showing that the incidence of seizures in patients with brain tumors is $30-70 \%[2,3]$.

Of this population, we collected 23 patients over a 4-year period, who developed RSE. Our study found that SE was terminated in $16 / 23(70 \%)$ patients with primary and metastatic brain tumors receiving combination therapy of PHT, LEV, and PGB (the Trifecta). Only 1/16 (6\%) patients who responded to the Trifecta required intubation. It is desirable to avoid intubation since sedation and intubation can result in prolonged length of stay and increased complication rates, especially in patients with cancer. We have shown here that this population of patients with RSE can be effectively managed without anesthetic doses of sedatives. In addition, we have shown a significant reduction in mortality. None of the patients who responded to the Trifecta died, which is an important point since the mortality from SE in patients with brain tumors has been shown to be $30-40 \%[7,11,12]$. Our overall mortality rate was $9 \%$.

Cerebral edema on imaging may be a marker for response to the Trifecta. Four patients in the responder group had peritumoral edema on MRI, compared to nil patients in the non-responder group, but this result was not statistically significant $(P=0.12)$. Even in the cases where a fourth AED was needed, control of seizures was obtained without anesthetic use in 19 of the 23 patients. After control of RSE is achieved, some of the AEDs can be weaned. The majority of patients were on AED monotherapy at 1 year follow-up, demonstrating that our patients were not condemned to high dose AEDs and poor cognitive function as an outcome of polypharmacy.

The significance of our data is limited by the retrospective, uncontrolled design and small sample size. More prospective studies with larger sample sizes are needed to confirm our results. However, to our knowledge there are no prior publications to guide therapy in this population of patients. Our results suggest that that the use of the Trifecta (PHT, LEV, and PGB) in brain tumor patients with RSE is safe and highly effective.

\section{References}

1. Porter KR, et al. Prevalence estimates for primary brain tumors in the United States by age, gender, behavior, and histology. Neuro Oncol. 2010;12(6):520-7.

2. Hauser WA, Annegers JF, Kurland LT. Incidence of epilepsy and unprovoked seizures in Rochester, Minnesota: 1935-1984. Epilepsia. 1993;34(3):453-68.

3. Morris $\mathrm{HH}$, et al. Chronic intractable epilepsy as the only symptom of primary brain tumor. Epilepsia. 1993;34(6):1038-43.

4. Hildebrand J, et al. Epileptic seizures during follow-up of patients treated for primary brain tumors. Neurology. 2005;65(2):212-5.

5. Moots PL, et al. The course of seizure disorders in patients with malignant gliomas. Arch Neurol. 1995;52(7):717-24.

6. Aminoff MJ, Simon RP. Status epilepticus. Causes, clinical features and consequences in 98 patients. Am J Med. 1980;69(5):657-66.

7. DeLorenzo RJ, et al. A prospective, population-based epidemiologic study of status epilepticus in Richmond, Virginia. Neurology. 1996;46(4):1029-35.

8. Knake $\mathrm{S}$, et al. Incidence of status epilepticus in adults in Germany: a prospective, population-based study. Epilepsia. 2001;42(6):714-8.

9. Sagduyu A, Tarlaci S, Sirin H. Generalized tonic-clonic status epilepticus: causes, treatment, complications and predictors of case fatality. J Neurol. 1998;245(10):640-6.

10. Claassen J, et al. Predictors of functional disability and mortality after status epilepticus. Neurology. 2002;58(1):139-42.

11. Towne AR, et al. Determinants of mortality in status epilepticus. Epilepsia. 1994;35(1):27-34.

12. Fountain NB. Status epilepticus: risk factors and complications. Epilepsia. 2000;41(Suppl 2):S23-30.

13. Claassen J, Hirsch LJ, Mayer SA. Treatment of status epilepticus: a survey of neurologists. J Neurol Sci. 2003;211(1-2):37-41.

14. Lowenstein DH, Alldredge BK. Status epilepticus. N Engl J Med. 1998;338(14):970-6.

15. Knake $S$, et al. Intravenous levetiracetam in the treatment of benzodiazepine refractory status epilepticus. J Neurol Neurosurg Psychiatry. 2008;79(5):588-9.

16. Moddel $\mathrm{G}$, et al. Intravenous levetiracetam: a new treatment alternative for refractory status epilepticus. J Neurol Neurosurg Psychiatry. 2009;80(6):689-92.

17. Rossetti AO, Bromfield EB. Levetiracetam in the treatment of status epilepticus in adults: a study of 13 episodes. Eur Neurol. 2005;54(1):34-8.

18. Gaitanis JN, Drislane FW. Status epilepticus: a review of different syndromes, their current evaluation, and treatment. Neurologist. 2003;9(2):61-76.

19. Novy J, Rossetti AO. Oral pregabalin as an add-on treatment for status epilepticus. Epilepsia. 2010;51(10):2207-10.

20. Ben-Menachem E. Pregabalin pharmacology and its relevance to clinical practice. Epilepsia. 2004;45(Suppl 6):13-8.

21. Cranford RE, et al. Intravenous phenytoin: clinical and pharmacokinetic aspects. Neurology. 1978;28(9 Pt 1):874-80.

22. Lynch BA, et al. The synaptic vesicle protein SV2A is the binding site for the antiepileptic drug levetiracetam. Proc Natl Acad Sci USA. 2004;101(26):9861-6. 